\title{
Donor Hypernatremia Influences Outcomes Following Pediatric Liver Transplantation
}

\author{
Neema Kaseje ${ }^{1}$ Samuel Lüthold ${ }^{2} \quad$ Gilles Mentha $^{3} \quad$ Christian Toso $^{3} \quad$ Dominique Belli $^{2}$ Valérie McLin ${ }^{2}$ \\ Barbara Wildhaber ${ }^{1}$ \\ ${ }^{1}$ Department of Pediatric Surgery, University Children's Hospital \\ Geneva, Geneva, Switzerland \\ 2 Department of Pediatrics, University Children's Hospital Geneva, \\ Geneva, Switzerland \\ ${ }^{3}$ Department of Surgery, University Hospital Geneva, Geneva, \\ Switzerland \\ Eur J Pediatr Surg 2013;23:8-13.

\begin{abstract}
Address for correspondence and reprint requests Barbara Wildhaber, MD, Department of Pediatric Surgery, University Children's Hospital Geneva, 6 rue Willy Donzé, Geneva 1211, Switzerland (e-mail: barbara.wildhaber@hcuge.ch).
\end{abstract}

\begin{abstract}
Introduction With the rising demand for liver transplantations (LTs), and the shortage of organs, extended criteria including donor hypernatremia have been adopted to increase the donor pool. Currently, there is conflicting evidence on the effect of donor hypernatremia on outcomes following LT. Our aim was to investigate differences in outcome in patients receiving grafts from hypernatremic donors compared with patients receiving grafts from normonatremic donors in the pediatric population.

Methods We retrospectively reviewed 94 pediatric patients with LTs from 1994 to 2011. We divided the patients into two groups: patients receiving organs from donors with sodium levels $<150 \mu \mathrm{mol} / \mathrm{L}, n=67$ (group 1 ), and patients receiving organs from donors with sodium levels $\geq 150 \mu \mathrm{mol} / \mathrm{L}, n=27$ (group 2). Using proportions and means, we analyzed patient age, sex, weight, model for end-stage liver disease (MELD) score, primary diagnosis, emergency of procedure, intraoperative transfusion volume, cold ischemia time, donor age, graft type, and postoperative graft function. Rates of mortality, rejection, early biliary, infectious, and vascular complications were calculated.

Results Mean age was 3.9 years in group 1 and 3.7 years in group $2(p=0.69)$. Mean weight and MELD scores were similar in the two study groups ( 16.0 vs. 15.9 and 21.2 vs. 22.0 , respectively). There were no significant differences in mean cold ischemia times 6.4 versus 6.9 hours $(p=0.29)$, and mean intraoperative transfusion volumes 1,068.5 $\mathrm{mL}$ versus $1,068.8 \mathrm{~mL}(p=0.89)$. There were no statistically significant differences in mortality rates ( 7.3 vs. $11.1 \%, p=0.68)$. Prothrombin time (PT) at day 10 post-LT was significantly lower in group 2 ( 79 vs. $64, p=0.017$ ), and there was a higher relative risk $(R R)$ for early thrombotic vascular complications in group $2(R R=2.48)$; however, this

Keywords

- hypernatremia

- pediatric liver transplantation

- outcomes was not significant $(p=0.26)$. No significant differences in RR for rejection $(0.97$, $p=0.86)$, viral infections $(1.24, p=0.31)$, bacterial infections $(0.86, p=0.62)$, or early biliary complications $(1.03, p=1.00)$ were observed.

Conclusion In pediatric LT patients receiving grafts from hypernatremic donors, there are no significant increases in rates of mortality, rejection, early biliary, and infectious
\end{abstract}

received

May 18, 2012

accepted after revision

August 18, 2012

published online

November 19, 2012 (c) 2013 Georg Thieme Verlag KG Stuttgart · New York
DOI http://dx.doi.org/ 10.1055/s-0032-1329703. ISSN 0939-7248. 
complications. However, there is a statistically significant lower PT at postoperative day 10 following transplantation, and a more than double RR for early thrombotic vascular complications although this was not statistically significant.

\section{Introduction}

Historically, liver transplant physicians have avoided using organs from hypernatremic donors for fear of poorer postoperative outcomes. However, the data supporting this assumption are currently limited, conflicted, and mainly address the adult population. Several authors have found no differences in survival or graft function following reception of grafts from hypernatremic donors. ${ }^{1,2}$ At the same time, other authors have found increased risk of early graft loss, poorer graft function, or even primary graft nonfunction. ${ }^{3-5,7,8}$

With the rising demand for liver transplantations (LTs), and the shortage of organs, extended criteria including donor hypernatremia have been adopted in many centers to increase the donor pool.

Our aim was to investigate differences in outcome in pediatric patients receiving grafts from hypernatremic donors compared with pediatric patients receiving grafts from donors with normal sodium levels. Our hypothesis was that there is no difference in outcome in patients receiving grafts from hypernatremic donors compared with patients receiving grafts from normonatremic donors.

\section{Methods}

\section{Patients and Setting}

We performed a retrospective chart review of 110 pediatric patients at the University Children's Hospital Geneva from the year 1994 to 2011, in whom LTs had been performed. The University Children's Hospital Geneva is the Swiss referral center for pediatric LTs with a specialized team of pediatric surgeons and pediatric hepatologists dedicated to pediatric LT patients.

Before 2000, most left lateral segments were reduced livers (ex situ reduction); only after 2000 were splits performed, most of them in situ. The LT operative procedure was similar all through the study period, with one and the same surgical team. Postoperative patient care varied slightly over time, but generally followed the same basic principles.

\section{Study Groups}

After excluding 16 patients without documented donor sodium levels, we divided the remaining 94 patients into two groups: group 1, patients receiving organs from donors with sodium levels less than $150 \mu \mathrm{mol} / \mathrm{L}(n=67)$, and group 2 , patients receiving organs from donors with sodium levels equal to or above $150 \mu \mathrm{mol} / \mathrm{L}(n=27)$. The donor sodium levels were final sodium values measured at the time of procurement which may or may not have been peak sodium levels.

\begin{abstract}
Analysis
We then analyzed potential differences between the two groups with regards to basic and operative characteristics as well as outcomes following pediatric LT. Basic characteristics examined included patient age, sex, mean model for end-stage liver disease (MELD) score, and primary diagnosis. Furthermore, we analyzed operative factors including emergency of procedure, cold ischemia time, and intraoperative blood transfusion volume. In addition, we examined donor age, and the type of graft that was used.
\end{abstract}

\section{Outcome Measures}

Our primary outcome measures were mortality within 1 year of LT and early graft function as indicated by postoperative liver function tests (aspartate transaminase [AST], alanine transaminase [ALT], and total bilirubin) and prothrombin time (PT) at 1, 3, 5, and 10 days after LT. Our secondary outcome measures were acute rejection, early biliary, infectious, and vascular complications. We defined early events as events occurring within the first 4 weeks of the LT.

\section{Statistical Analysis}

Using proportions for categorical data and means and standard deviations for continuous data, we analyzed patient age, sex, primary diagnosis, MELD score, emergency of procedure, cold ischemia time, intraoperative transfusion volume, donor age, graft type, and postoperative graft function. In addition, we calculated rates of mortality, acute rejection, early biliary, infectious, and vascular complications. Significance was determined using the chi-square, the Fisher exact, and the Mann-Whitney tests. A $p$ value of $<0.05$ was considered significant.

Ethical approval for this study was obtained from the Ethics Committee (CER 11-01OR/MATPED 11-004R).

\section{Results}

Analysis of the descriptive characteristics of the two study populations revealed a higher proportion of female patients in group 1 compared with group 2; mean age in years at LT was slightly higher in group 1 (-Table 1); and the mean weight and MELD scores were almost identical in the two groups (-Table 1). A higher proportion of patients in group 1 had the primary diagnosis of biliary atresia ( - Table 1 ). The $p$ value column of - Table 1 summarizes the fact that there were no statistically significant differences in the basic characteristics of the two study populations.

Examination of operative factors demonstrated differences in the proportion of emergent procedures and mean cold ischemia times between group 1 and group 2 that were not 
Table 1 Comparison of descriptive patient characteristics: there were no statistically significant differences in basic patient characteristics between the two study populations

\begin{tabular}{|l|l|l|l|}
\hline Basic patient characteristic & $\begin{array}{l}\text { Group 1 } \\
(\mathbf{N a}<\mathbf{1 5 0} \mu \mathrm{mol} / \mathrm{L}) \\
\boldsymbol{n}=\mathbf{6 7}\end{array}$ & $\begin{array}{l}\text { Group 2 } \\
(\mathbf{N a} \geq \mathbf{1 5 0} \boldsymbol{\mu m o l} / \mathrm{L}) \\
\boldsymbol{n}=\mathbf{2 7}\end{array}$ & $p$ value $^{\mathbf{a}}$ \\
\hline Male (\%) & 47.8 & 51.8 & 0.71 \\
\hline Female (\%) & 55.2 & 48.2 & 0.53 \\
\hline Mean age at LT (years) & 3.9 & 3.7 & 0.69 \\
\hline Mean weight (kg) & 16.0 & 15.9 & 0.69 \\
\hline Mean MELD score & 21.2 & 22.0 & 0.89 \\
\hline Primary diagnosis biliary atresia (\%) & 50.7 & 40.7 & 0.38 \\
\hline
\end{tabular}

Abbreviations: LT, liver transplantation; MELD, model for end-stage liver disease; Na, sodium.

${ }^{a}$ Chi-square test for sex and primary diagnosis biliary atresia; Mann-Whitney test for age, weight, and MELD score.

statistically significant ( $\sim$ Table 2 ). Mean transfusion volume was essentially the same in the two groups (-Table 2). Mean donor age, proportion of whole, reduced, and split grafts were also similar between the two groups (-Table 2 ).

In analyzing our primary outcome measures, we found a higher mortality rate within 1 year of LT in group 2 compared with group 1 ; however, this difference was not statistically significant ( - Table 3 ). We also found a higher proportion of primary nongraft function as a cause of death in group 2 compared with group 1 (-Table 3 ). Looking at primary graft function as illustrated by postoperative changes in liver function tests, we saw a trend toward increased liver enzymes (AST and ALT) in postoperative days 1, 3, and 5 in group 2; however, this trend was not statistically significant ( - Table 3 ). The trend toward higher liver function tests in group 2 was not observed in the postoperative total bilirubin levels which were lower in group 2 compared with group 1 levels; this difference again was not statistically significant ( - Table 3 ). There was a consistently lower PT in group 2 compared with group 1 at postoperative days $1,3,5$, and 10 (-Table 3). This difference in PT was statistically significant at postoperative day 10 with a $p$ value of 0.017 . The results on differences in postoperative PTs are graphically shown in -Fig. 1; here we see that group 1 has increased PT with each subsequent postoperative day. Contrary to group 1, group 2 postoperative PTs remain persistently low (-Fig. 1).

Examination of our secondary outcome measures demonstrated no statistically significant differences in the relative risks (RRs) of acute rejection, early biliary complications, and infectious complications between the two study groups ( - Table 4). The RR for early thrombotic vascular complications was more than double in group 2 compared with group 1; however, this was not statistically significant ( - Table 4). A stratified analysis of early thrombotic vascular complications demonstrated a higher incidence of portal vein thrombosis in group 2 compared with group 1 ( - Table 5 ). In addition, there was a shorter mean time to thrombotic vascular complication in group 2 compared with group 1 (-Table 5).

\section{Discussion}

In this study, our goal was to evaluate the differences in outcome in pediatric patients receiving grafts from hypernatremic donors compared with pediatric patients receiving grafts from normonatremic donors.

To our knowledge, no such study has ever been performed in a pediatric cohort. In the adult LT literature, a relationship between donor hypernatremia and poorer outcomes

Table 2 Comparison of operative factors: there were no statistically significant differences in operative factors between the two study populations

\begin{tabular}{|c|c|c|c|}
\hline Operative factor & $\begin{array}{l}\text { Group } 1 \\
\text { (Na }<150 \mu \mathrm{mol} / \mathrm{L}) \\
n=67\end{array}$ & $\begin{array}{l}\text { Group } 2 \\
\text { (Na } \geq 150 \mu \mathrm{mol} / \mathrm{L}) \\
n=27\end{array}$ & $p$ value $^{a}$ \\
\hline Emergent procedure (\%) & 13.4 & 25.9 & 0.14 \\
\hline Mean cold ischemia time (hours) & 6.4 & 6.9 & 0.29 \\
\hline Mean transfusion (mL) & $1,068.5$ & $1,068.8$ & 0.89 \\
\hline Mean donor age in years (pediatric donors \%) & $22.7(34.8)$ & $20.9(33.3)$ & 0.60 \\
\hline Whole liver (\%) & 34.8 & 33.3 & 0.88 \\
\hline Reduced and split liver (\%) & 65.2 & 66.7 & 0.89 \\
\hline
\end{tabular}

Abbreviation: Na, sodium.

${ }^{a}$ Chi-square test for emergent procedure and graft type; Mann-Whitney test for cold ischemia time, transfusion volume, and donor age. 
Table 3 Donor hypernatremia and primary outcome measures: mortality and primary graft function

\begin{tabular}{|c|c|c|c|}
\hline Outcome measure & $\begin{array}{l}\text { Group } 1 \\
(\mathrm{Na}<150 \mu \mathrm{mol} / \mathrm{L}) \\
n=67\end{array}$ & $\begin{array}{l}\text { Group } 2 \\
(\mathrm{Na} \geq 150 \mu \mathrm{mol} / \mathrm{L}) \\
n=27\end{array}$ & $p$ value $^{\mathrm{a}}$ \\
\hline Mortality (\%) & 7.3 & 11.1 & 0.68 \\
\hline Mortality due to primary nongraft function (\%) & 40 & 67 & 0.57 \\
\hline AST_D1 IU/L & 965 & 1,267 & 0.18 \\
\hline AST_D3 IU/L & 294 & 309 & 0.35 \\
\hline AST_D5 IU/L & 84 & 147 & 0.53 \\
\hline AST_D10 IU/L & 67 & 56 & 0.22 \\
\hline ALT_D1 IU/L & 712 & 739 & 0.62 \\
\hline ALT_D3 IU/L & 502 & 644 & 0.67 \\
\hline ALT_D5 IU/L & 249 & 302 & 0.57 \\
\hline ALT_D10 IU/L & 149 & 98 & 0.14 \\
\hline BILI TOT_D1 $\mu \mathrm{mol} / \mathrm{L}$ & 135 & 115 & 0.79 \\
\hline BILI TOT_D3 $\mu \mathrm{mol} / \mathrm{L}$ & 106 & 92 & 0.32 \\
\hline BILI TOT_D5 $\mu \mathrm{mol} / \mathrm{L}$ & 87 & 72 & 0.35 \\
\hline BILI TOT_D10 $\mu \mathrm{mol} / \mathrm{L}$ & 71 & 59 & 0.10 \\
\hline PT\%_D1 & 59 & 56 & 0.56 \\
\hline PT\%_D3 & 75 & 63 & 0.09 \\
\hline PT\%_D5 & 71 & 62 & 0.15 \\
\hline PT\%_D10 & 79 & 64 & 0.017 \\
\hline
\end{tabular}

Abbreviations: ALT, alanine transaminase; AST, aspartate transaminase; BILI TOT, total bilirubin; D, postoperative day; Na, sodium; PT, prothrombin time.

Note: Group 1 ( $\mathrm{Na}<150 \mu \mathrm{mol} / \mathrm{L}$ ) has increased PT with each subsequent postoperative day, whereas group 2 (Na $\geq 150 \mu \mathrm{mol} / \mathrm{L}$ ) postoperative PTs remain persistently low.

${ }^{a}$ Fisher exact test for mortality and mortality due to primary nongraft function; Mann-Whitney test for liver function tests and PT.

following LT was first suggested by Avolio et al in $1991 .^{8}$ This study was later followed by González et al's study where they analyzed 54 variables to identify factors predictive of early postoperative graft function. ${ }^{5}$ In their analysis, they found

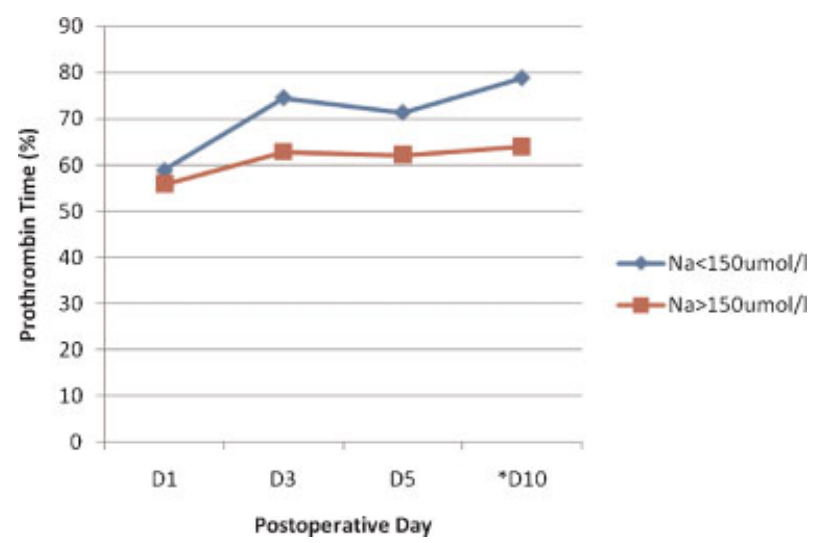

Fig. 1 Differences in changes in early postoperative prothrombin time. Note the significant difference between group $1(\mathrm{Na}<150 \mu \mathrm{mol} / \mathrm{L})$ and group 2 ( $\mathrm{Na} \geq 150 \mu \mathrm{mol} / \mathrm{L}$ ) prothrombin time at day 10 after LT. ${ }^{*} p=0.017 ; \mathrm{D}$, postoperative day; Na, sodium. that donor sodium greater than $155 \mu \mathrm{mol} / \mathrm{L}$ was a predictor of graft dysfunction. ${ }^{5}$ Similar conclusions were made by Figueras et al who found that sodium greater than $155 \mu \mathrm{mol} / \mathrm{L}$ was independently associated with graft loss within a month postLT. $^{6}$ In addition, Totsuka et al found that donor sodium greater than $155 \mu \mathrm{mol} / \mathrm{L}$ was associated with increased liver function tests, prolonged coagulation, and increased graft loss. ${ }^{3,4}$ However, in more recent studies, Cywinski et al found no relation between donor hypernatremia (sodium $\geq 155 \mu \mathrm{mol} / \mathrm{L}$ ) and poor outcomes following LT. ${ }^{1}$ Similarly, Mangus et al found that donor sodium greater than $160 \mu \mathrm{mol} / \mathrm{L}$ had no impact on recipient graft function and survival. ${ }^{2}$ In our study, we chose to define significant hypernatremia as sodium equal to or greater than $150 \mu \mathrm{mol} / \mathrm{L}$ based on previous studies; the majority of which defined significant hypernatremia as sodium greater than $155 \mu \mathrm{mol} / \mathrm{L}$; and based on our institutional cutoff for hypernatremia of $143 \mu \mathrm{mol} / \mathrm{L}^{2-4,6}$

In comparing basic characteristics between our two study populations, we observed no statistically significant differences in age, sex, weight, and MELD score. In addition, there were no statistically significant differences in operative characteristics of cold ischemia time, percentage of emergency procedure, volume of intraoperative transfusion, donor age, 
Table 4 Donor hypernatremia and secondary outcome measures: there is no statistically significant increase in relative risk of acute rejection, early biliary, and infectious complications in group 2 ( $\mathrm{Na} \geq 150 \mu \mathrm{mol} / \mathrm{L}$ ) compared with group 1 ( $\mathrm{Na}<150 \mu \mathrm{mol} / \mathrm{L}$ )

\begin{tabular}{|c|c|c|c|c|}
\hline Outcome measure & $\begin{array}{l}\text { Group } 1 \\
(\mathrm{Na}<150 \mu \mathrm{mol} / \mathrm{L}) \\
n=67\end{array}$ & $\begin{array}{l}\text { Group } 2 \\
(\mathrm{Na} \geq 150 \mu \mathrm{mol} / \mathrm{L}) \\
n=27\end{array}$ & Relative risk & $p$ value $^{a}$ \\
\hline Acute rejection (\%) & 61.2 & 59.3 & 0.97 & 0.86 \\
\hline Early biliary complications (\%) & 17.9 & 18.5 & 1.03 & 1.00 \\
\hline Bacterial infections (\%) & 38.8 & 33.3 & 0.86 & 0.62 \\
\hline Viral infections (\%) & 47.7 & 59.3 & 1.24 & 0.31 \\
\hline Early thrombotic vascular complications (\%) & 5.9 & 14.8 & 2.48 & 0.26 \\
\hline
\end{tabular}

Abbreviations: LT, liver transplantation; Na, sodium.

Note: The relative risk of early ( $<4$ weeks after LT) thrombotic vascular complications is more than double in group 2 (Na $\geq 150 \mu \mathrm{mol} / \mathrm{L}$ ) compared with group 1 ( $\mathrm{Na}<150 \mu \mathrm{mol} / \mathrm{L})$; this result however did not reach statistical significance.

${ }^{a}$ Chi-square test for all except early biliary and vascular complications for which the Fisher exact test was used.

Table 5 Donor hypernatremia and secondary outcome measures: stratified analysis of early thrombotic vascular complications $(<4$ weeks after LT)

\begin{tabular}{|l|l|l|}
\hline $\begin{array}{l}\text { Early thrombotic vascular complication } \\
(<4 \text { weeks after } \mathrm{LT})\end{array}$ & $\begin{array}{l}\text { Group } 1 \\
(\mathrm{Na}<150 \mu \mathrm{mol} / \mathrm{L}) \\
n=67\end{array}$ & $\begin{array}{l}\text { Group } 2 \\
(\mathrm{Na} \geq \mathbf{1 5 0} \mu \mathrm{mol} / \mathrm{L}) \\
n=27\end{array}$ \\
\hline Hepatic artery thrombosis & $4(6 \%)$ & $2(7 \%)$ \\
\hline Portal vein thrombosis & $0(0 \%)$ & $2(7 \%)$ \\
\hline Mean time to complication (days) & 9 & 5 \\
\hline
\end{tabular}

Abbreviations: LT, liver transplantation; Na, sodium.

Note: There is a higher incidence of portal vein thrombosis in group 2 (Na $\geq 150 \mu \mathrm{mol} / \mathrm{L}$ ) compared with group 1 (Na $<150 \mu \mathrm{mol} / \mathrm{L}$ ). There is a shorter mean time to thrombotic complication in group 2 ( $\mathrm{Na} \geq 150 \mu \mathrm{mol} / \mathrm{L})$ compared with group 1 ( $\mathrm{Na}<150 \mu \mathrm{mol} / \mathrm{L}$ ).

and graft type. Therefore, in our opinion, our two study populations were comparable and as a result, we could safely compare postoperative outcomes in the two groups.

In reviewing the primary outcome measures, we found a higher mortality rate within 1 year of LT in children having received livers from hypernatremic donors compared with those receiving grafts from normonatremic donors (11.1 vs. 7.3\%); this finding however was not statistically significant. The observation of no statistically significant difference in mortality between the two groups is corroborated by Cywinski et al. ${ }^{1}$ In their study, Cywinski et al evaluated the relationship between donor-recipient sodium differences and adult liver transplant graft function and survival; they concluded that donor hypernatremia did not affect patient survival at 30 days and at 1 year. ${ }^{1}$ A subanalysis of our deaths revealed that a higher proportion of group 2 patients died secondary to primary graft nonfunction compared with group 1 (67 vs. $40 \%$ ); this observation was not statistically significant, but one could still make the argument that donor hypernatremia may be associated with primary nongraft function and subsequent death. A larger study should be performed in the pediatric population to further explore the relationship between donor hypernatremia and primary graft nonfunction with subsequent death. In the adult LT literature, Totsuka et al found that donor hypernatremia $>155 \mu \mathrm{mol} / \mathrm{L}$ was associat- ed with early graft loss. ${ }^{3,4}$ When we looked at mortality beyond 1 year after LT, we found that our mortality rates of $7.3 \%$ for group 1 and $11.1 \%$ for group 2 remained the same 5 years after LT. We can deduce therefore that factors during the perioperative period including donor hypernatremia were important in determining survival of our patients. Four out of the total eight deaths in both groups occurred within 4 days of LT; six out of the eight deaths occurred within a month of LT.

Regarding differences in primary graft function, there was a trend toward increased ALT and AST in the early postoperative days 1,3 , and 5 in group $2(\mathrm{Na} \geq 150 \mu \mathrm{mol} / \mathrm{L})$; this observation again was not statistically significant. It is quite possible that with larger numbers of subjects within each study group, we may have identified a statistically significant increase in liver enzyme values, hence deduced that donor hypernatremia is related to poorer graft function in the early postoperative period.

In reviewing the literature regarding primary graft function and donor hypernatremia, Cywinski et al, found no association between donor hypernatremia and immediate postoperative AST, ALT, and bilirubin on postoperative days 1 , 2 , and 3 in adult liver transplant patients. ${ }^{1}$ Totsuka et al however found increased postoperative liver enzymes in patients receiving grafts from hypernatremic donors. ${ }^{4}$ 
Of interest, we observed a persistently lower PT (which translates into prolonged coagulation time) in the early postoperative period in the hypernatremic donor group compared with the normonatremic donor group. This difference in coagulation time between the two study groups was statistically significant on postoperative day 10 . We found no studies in the pediatric literature analyzing the effect of hypernatremia on postoperative coagulation following LT. In the adult literature, similar results were observed by Totsuka et al who demonstrated prolonged coagulation time in patients receiving grafts from donors with sodium levels greater than $155 \mu \mathrm{mol} / \mathrm{L}^{4}$

There is no clear explanation for the association of donor hypernatremia and poorer graft function as illustrated by a trend toward increased liver enzymes and prolonged coagulation times in the early postoperative period. However, the current hypothesis for early graft dysfunction related to donor hypernatremia is that differences in osmolarity between the graft hepatocellular cells and the recipient may result in movement of water into the hepatocellular cells with subsequent edema and hepatocellular cell dysfunction. ${ }^{5}$

Examination of our secondary outcome measures revealed increased vascular complications in patients receiving grafts from hypernatremic donors. In reviewing the literature, we found no study associating donor hypernatremia with increased vascular complications in either the pediatric or adult LT literature. Although the RR for early thrombotic vascular complications was more than double in the hypernatremic donor group compared with the normonatremic donor group; due to a low incidence rate in both study groups, this observation was not statistically significant. A potential explanation for the increased early thrombotic vascular complications in the hypernatremic donor group is that hepatocellular edema that results from grafts coming from hypernatremic donors might result in stasis of vascular flow and subsequent thrombosis. There were no statistically significant differences in early biliary complications, infection risk, and rejection risk in patients receiving grafts from hypernatremic donors. In reviewing the literature, we found no other studies looking at the effect of donor hypernatremia and these factors.

There were several limitations to our study. First, it was a retrospective review, and there were missing data on 16 patients for which we did not have documented donor sodium levels. Second, our study population was small, therefore, we may not have identified differences that may be present but for which our study population was not large enough to detect. Furthermore, there were likely changes in practice in terms of postoperative management of patients that likely introduced some level of bias that was difficult to measure and to adjust for in our analyses.

Our study had several advantages: we examined pediatric patients alone which to our knowledge has not been previously done. Furthermore, we looked at outcome measures not previously examined by other authors including early vascular, biliary, infectious complications, and rejection, and how these are influenced by donor hypernatremia.

In conclusion, in analyzing pediatric liver transplant patients receiving grafts from hypernatremic donors, there were no statistically significant increases in mortality, acute rejection, early biliary, and infectious complications. However, we observed a statistically significant lower PT at day 10 following transplantation. In addition, we observed a more than double RR for early thrombotic vascular complications; this result, however, did not reach statistical significance. A larger study is needed to further evaluate the effect of donor hypernatremia on outcomes following pediatric LT.

Conflict of Interest

None

\section{References}

1 Cywinski JB, Mascha E, Miller C, et al. Association between donorrecipient serum sodium differences and orthotopic liver transplant graft function. Liver Transpl 2008;14(1):59-65

2 Mangus RS, Fridell JA, Vianna RM, et al. Severe hypernatremia in deceased liver donors does not impact early transplant outcome. Transplantation 2010;90(4):438-443

3 Totsuka E, Fung U, Hakamada K, et al. Analysis of clinical variables of donors and recipients with respect to short-term graft outcome in human liver transplantation. Transplant Proc 2004;36(8):2215-2218

4 Totsuka E, Dodson F, Urakami A, et al. Influence of high donor serum sodium levels on early postoperative graft function in human liver transplantation: effect of correction of donor hypernatremia. Liver Transpl Surg 1999;5(5):421-428

5 González FX, Rimola A, Grande L, et al. Predictive factors of early postoperative graft function in human liver transplantation. Hepatology 1994;20(3):565-573

6 Figueras J, Busquets J, Grande L, et al. The deleterious effect of donor high plasma sodium and extended preservation in liver transplantation. A multivariate analysis. Transplantation 1996;61 (3):410-413

7 Briceno J, Lopez-Cillero P, Rufian S, et al. Impact of marginal quality donors on the outcome of liver transplantation. Transplant Proc 1997;29(1-2):477-480

8 Avolio AW, Agnes S, Magalini SC, Foco M, Castagneto M. Importance of donor blood chemistry data (AST, serum sodium) in predicting liver transplant outcome. Transplant Proc 1991; 23(5):2451-2452 